\title{
General
}

\section{Thoracic Surgery}

\section{Stage III non-small cell lung cancer and metachronous brain metastases}

Nader Moazami, MD

Thomas W. Rice, $\mathrm{MD}^{\mathrm{a}}$

Lisa A. Rybicki, MS ${ }^{\text {b }}$

David J. Adelstein, MD ${ }^{\mathrm{c}}$

Sudish C. Murthy, MD, $\mathrm{PhD}^{\mathrm{a}}$

Malcolm M. DeCamp, MD

Gene H. Barnett, MD ${ }^{\mathrm{d}}$

Mark A. Chidel, MD

John H. Suh, MD

Eugene $\mathrm{H}$. Blackstone, MD ${ }^{\mathrm{a}, \mathrm{b}}$

Objectives: This study was undertaken to identify management strategies that maximize survival of patients with stage III non-small cell lung cancer and metachronous brain metastases and to determine whether any apparent improved survival was due to treatment or simply to patient selection.

Methods: Treatment evaluations of both primary non-small cell lung cancer and brain metastases were performed in 91 patients. Optimal treatment was identified by multivariable analysis. Propensity scoring and multivariable analysis were used to separate treatment benefit from patient selection.

From the Departments of Thoracic and Cardiovascular Surgery, ${ }^{\text {a }}$ Biostatistics and Epi-

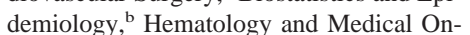
cology, ${ }^{\mathrm{c}}$ Neurological Surgery, ${ }^{\mathrm{d}}$ and Radiation Oncology, ${ }^{\mathrm{e}}$ The Cleveland Clinic Foundation, Cleveland, Ohio

Read at the Eighty-first Annual Meeting of The American Association for Thoracic Surgery, San Diego, Calif, May 6-9, 2001.

Received for publication April 27, 2001; revisions requested July 10, 2001; revisions received Sept 28, 2001; accepted for publication Nov 9, 2001

Address for reprints: Thomas W. Rice, MD, The Cleveland Clinic Foundation, 9500 Euclid Ave, Desk F25, Cleveland, OH 44195 (E-mail: ricet@ccf.org).

J Thorac Cardiovasc Surg 2002;124:113-22

Copyright ( $(2) 2002$ by The American Association for Thoracic Surgery

0022-5223/2002 \$35.00+0 $\quad \mathbf{1 2 / 6 / 1 2 1 6 7 8}$

doi:10.1067/mtc.2002.121678

Results: Risk-unadjusted median, 12-, and 24-month survivals were 5.2 months, $22 \%$, and $10 \%$, respectively. Younger age $(P=.006)$, good performance status $(P=.003)$, stage IIIA $(P=.001)$, lung resection $(P=.02)$, no other systemic metastases at time of diagnosis of brain metastases $(P=.02)$, and either metastasectomy $(P<.001)$ or stereotactic radiosurgery $(P<.001)$ predicted best survival. However, metastasectomy or stereotactic radiosurgery was more common after lung resection $(P=.02)$ and in patients with good performance status $(P=.006)$, no other systemic metastases at time of diagnosis of brain metastases $(P=.01)$, and fewer brain metastases $(P<.001)$, suggesting that the patients with the best risk profile were selected for aggressive therapy of both lung primary and brain metastases. Despite this selection, analysis of propensity-matched patients demonstrated the benefit of lung resection and metastasectomy or stereotactic radiosurgery $(P<.001)$.

Conclusions: Younger patients with resected stage IIIA non-small cell lung cancer who have isolated metachronous brain metastases and good performance status do best when treated with metastasectomy or stereotactic radiosurgery. This survival benefit is a brain treatment effect, not the result of selecting the best patients for aggressive therapy. 


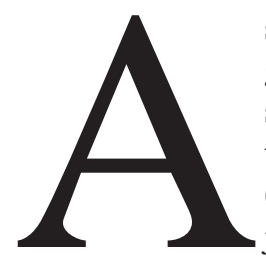

s early as the 1970s, clinical reports suggested that aggressive therapy provided a survival advantage for highly selected patients with non-small cell lung cancer (NSCLC) and brain metastases..$^{1,2}$ Neoadjuvant therapy for stage III NSCLC has produced a unique cohort of patients who have survived to have metachronous brain metastases develop. ${ }^{3}$ Optimal management in these cases is uncertain, ${ }^{4}$ a difficulty that provided the impetus for this study. The purposes of this study were to identify management strategies that maximize survival of patients with stage III NSCLC and metachronous brain metastases and to determine whether any apparent improved survival was due to treatment or simply patient selection.

\section{Patients and Methods}

\section{Patients}

From review of the Cleveland Clinic Foundation hospital discharge database, Cancer Center Registry, and Radiation Oncology Registry, 91 patients were identified with metachronous brain metastases after a diagnosis of stage IIIA or IIIB NSCLC. Metachronous brain metastasis was considered to be a brain metastasis occurring 3 or more months after diagnosis of the primary NSCLC. Brain metastases were confirmed by computed tomography, magnetic resonance imaging, or both. Stage IIIA or IIIB NSCLC was Tany N2 M0, Tany N3 M0, or T4 Nany M0 (excluding malignant pleural effusions and separate tumor nodules in the same lobe) documented by mediastinoscopy $(n=48)$, thoracotomy $(n=9)$, or computed tomography $(\mathrm{n}=34)$.

Cross-sectional systematic follow-up was done in February 2001. Eighty-three patients were dead. Mean follow-up was 36 months (range 2.5 to 96 months) for 8 living patients. Time zero for time-related analysis was measured from diagnosis of metachronous brain metastases. All-cause mortality was the study end point.

\section{Data Analysis}

Identification of optimal management. The goal of assessing management was identification of strategies that maximized survival. Nonparametric estimates of survival after development of brain metastases were obtained using the Kaplan-Meier method. Parametric estimates of survival were obtained by hazard function analysis. ${ }^{5}$ Survival estimates were compared between subgroups using the log-rank test.

Both multivariable Cox proportional hazards analysis and parametric hazard function analysis of all variables listed in Appendix Table A were used to identify risk factors for death after development of brain metastases. A stepwise selection procedure was used, with a $P=.05$ criterion for retention of variables in the final model. This procedure was verified by bootstrap bagging with 1000 samples. ${ }^{6}$ Variables identified in at least $50 \%$ of the bootstrap analyses were considered reliable. Continuous and ordinal variables were assessed univariably by decile risk analysis to determine whether their relationship was well calibrated to model outcome or whether transformations of scale were required.
Treatment benefit or patient selection? Because there was no treatment protocol, patient selection itself could lead to spurious conclusions that certain therapies improved survival. At the same time, the resulting variance in management permitted separation of treatment effect from that of patient selection by means of propensity score matching. ${ }^{7}$ The propensity model included 10 variables: gender, histologic type, stage at treatment of NSCLC, lung resection (vs none) for NSCLC, disease-free interval, number of brain metastases, local recurrence of NSCLC, presence of distant metastases other than in the brain, Eastern Cooperative Oncology Group (ECOG) performance status at diagnosis of brain metastases, and a recursive partitioning analysis (RPA) score. (RPA score ranges from 1 to 3 and is composed of age, performance status, recurrence of NSCLC, and presence of other distant metastases. ${ }^{8}$ ) All these variables were entered into a multivariable logistic regression model of metastasectomy or stereotactic radiosurgery versus palliation. For each patient, the model was used to calculate the probability of undergoing metastasectomy or stereotactic radiosurgery. This probability is the propensity score.

The propensity score was used in two ways to separate treatment from selection effects. First, we used it to compare survival benefit of metastasectomy or stereotactic radiosurgery within propensity-matched groups. For this, patients were sorted according to the propensity score and divided into two equal-sized groups. We then determined the effect of metastasectomy or stereotactic radiosurgery on survival within each of the groups using the Kaplan-Meier method and log-rank test. To assess similarity within each group, $t$ tests and contingency tables were used to compare patient factors, tumor factors, and treatment of NSCLC between patients who underwent metastasectomy or stereotactic radiosurgery and those who did not.

Second, the propensity score was used to confirm the results of the multivariable analysis that metastasectomy or stereotactic radiotherapy was beneficial. ${ }^{9}$ For this, the propensity score was incorporated into the multivariable survival model of optimal management, and its adjustment of treatment effect magnitude and statistical significance was observed.

Presentation. Hazard and odds ratios are accompanied by $95 \%$ confidence intervals (CIs). Depiction of the ideal candidate for metastasectomy or stereotactic radiosurgery and the ideal candidate for palliation of brain metastases used the hazard function analysis. Specific values were entered into the multivariable equation, the equation was solved, and results were presented graphically with $68 \%$ CIs equivalent to $1 \mathrm{SE}$.

\section{Results}

\section{Identification of Optimal Management}

Characterization and treatment of NSCLC. Fifty-two patients (57\%) had stage IIIA NSCLC. Tumor histologic type was squamous cell carcinoma in 25 patients (27\%), adenocarcinoma in $50(55 \%)$, and large cell carcinoma in 16 $(18 \%)$. NSCLC was not treated in 3 patients (3\%) because of treatment refusal in 1 patient and prohibitive comorbidities in 2. Treatment was chemoradiation therapy only in 29 patients (32\%). Surgery was used in 59 patients (65\%): resection in $11(12 \%)$, resection and postoperative adjuvant therapy in $22(24 \%)$, and neoadjuvant therapy (chemoradia- 


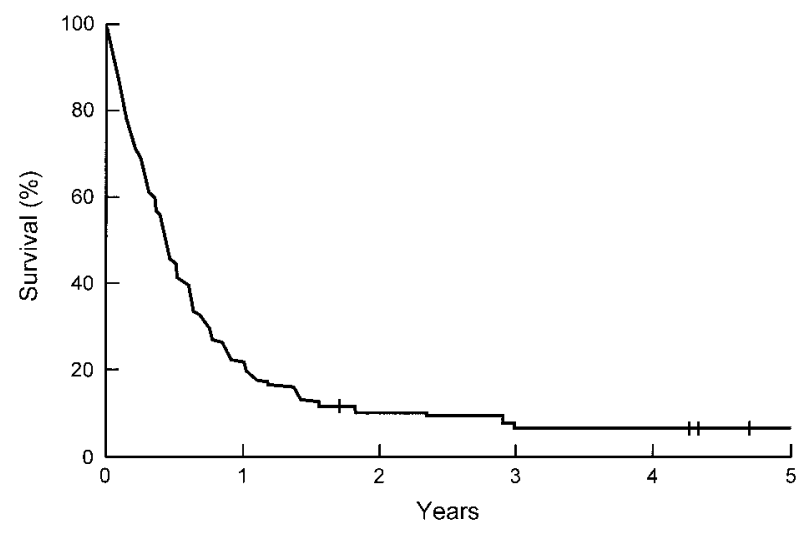

Figure 1. Risk-unadjusted survival. Vertical bars represent patients alive at last follow-up. Survival estimates \pm SE and numbers of patients remaining at risk at 6, 12, and 24 months were $45 \% \pm 5.3 \%(n=40), 22 \% \pm 4.4 \%(n=19)$, and $10 \% \pm 3.3 \%(n=$ 8), respectively.

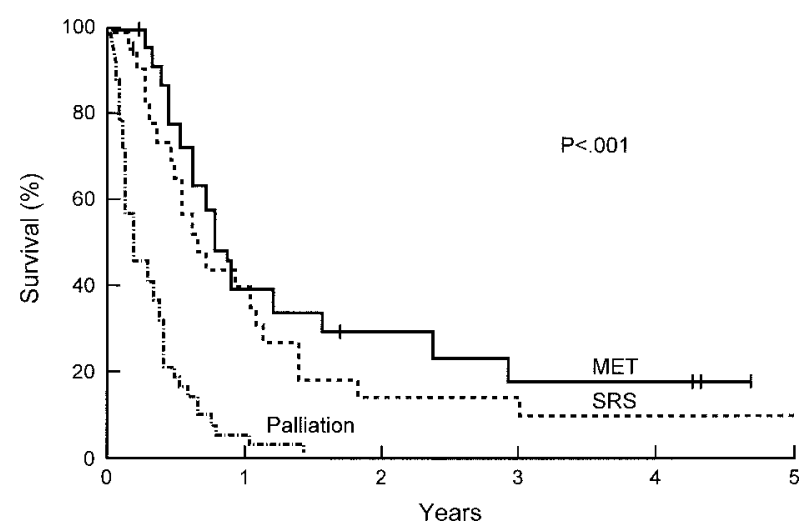

Figure 2. Survival according to treatment of metachronous brain metastases. Figure format as in Figure 1. Survival estimates \pm SE and numbers of patients remaining at risk at 6, 12, and 24 months were as follows: for metastasectomy (MET, solid line, $\mathrm{n}=23$ ), $77 \% \pm 8.9 \%(n=17), 39 \% \pm 10.6 \%(n=8)$, and $29 \% \pm 10.0 \%(n=$ 6), respectively; for stereotactic radiosurgery (SRS, dashed line, $n=24), 65 \% \pm 9.9 \%(n=15), 39 \% \pm 10.2 \%(n=9)$, and $13 \% \pm$ $7.0 \%$ ( $n=3$ ), respectively; and for palliation (unevenly broken line, $n=44), 18 \% \pm 5.8 \%(n=8), 5 \% \pm 3.1 \%(n=2)$, and $0 \%(n=$ $0)$, respectively.

tion and surgery) in $26(28 \%)$. Resection was segmentectomy in 2 patients (2\%), lobectomy in 35 patients $(38 \%)$, and pneumonectomy in 22 patients $(24 \%)$.

Characterization and treatment of brain metastases. Forty-two patients $(46 \%)$ had a single brain metastasis, 15 (16\%) had 2 metastases, 16 (18\%) had 3, and 18 (20\%) had 4 or more. Sixty-two patients $(68 \%)$ had unilateral brain metastases. Treatment of brain metastases was whole-brain radiation therapy in 43 patients (47\%), metastasectomy
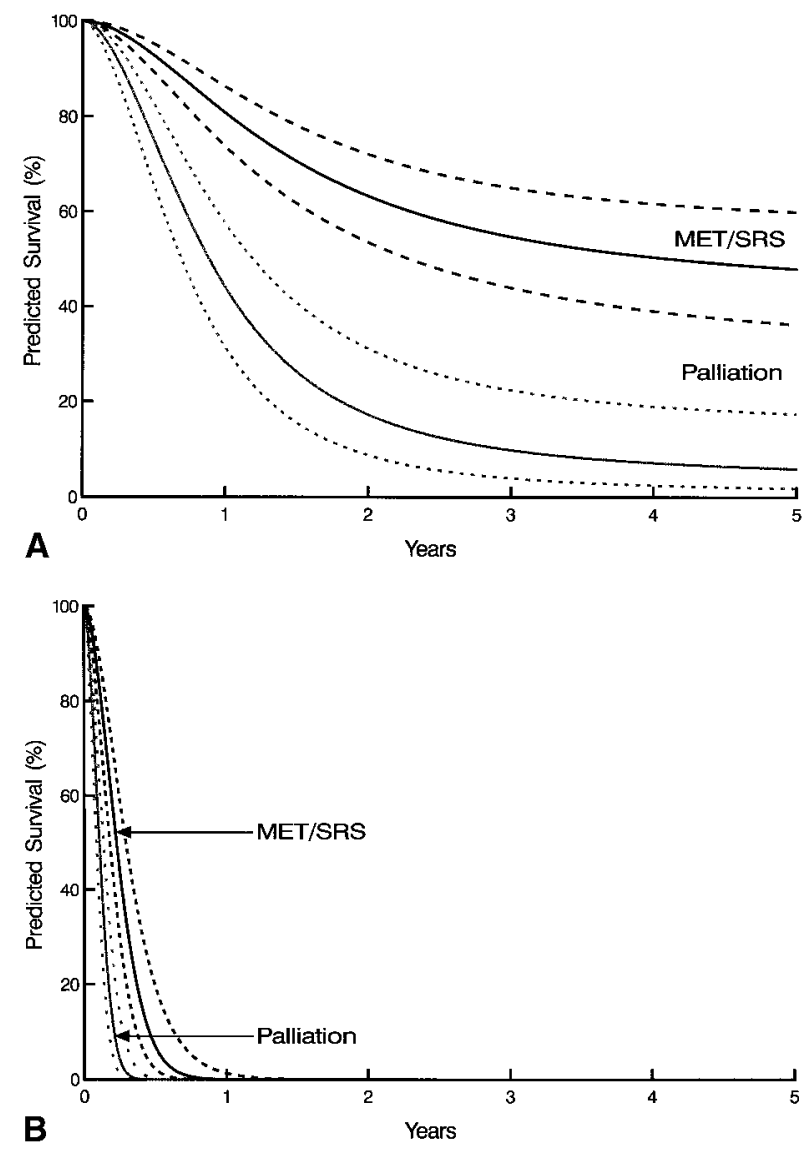

Figure 3. Comparison of metastasectomy or stereotactic radiosurgery (MET/SRS) with palliation in patients with characteristics predictive of good survival (A) and poor survival (B). A, 45-yearold patient with stage IIIA NSCLC treated by pneumonectomy. At diagnosis of metachronous brain metastases, this patient has ECOG 0 to 1 status with no other systemic metastases. B, 65-yearold patient with stage IIIB NSCLC not resected. At diagnosis of metachronous brain metastases, this patient has ECOG 2 status with other systemic metastases.

alone or in combination with whole-brain radiation therapy in 23 patients $(25 \%)$, and stereotactic radiosurgery with or without whole-brain radiation therapy in 24 patients $(26 \%)$. One patient received no treatment.

The impact of stage III NSCLC and its treatment on metachronous brain metastases is described in Appendix A.

Optimal management. Risk-unadjusted survivals at 3, 6,12 , and 24 months after diagnosis of metachronous brain metastases were $70 \%, 45 \%, 22 \%$, and $10 \%$, respectively, with a median survival of 5.2 months (Figure 1). Risk factors for death are listed in Table 1. Good survival was predicted by stage IIIA, resection of NSCLC, younger age at diagnosis of brain metastases, good performance status, no other distant metastases, and metastasectomy or stereotactic radiosurgery (Figure 2 and Appendix Figure A). 
TABLE 1. Risk factors for death after diagnosis of brain metastases

\begin{tabular}{|c|c|c|c|}
\hline Risk factor & Coefficient & $P$ value & Reliability* \\
\hline \multicolumn{4}{|l|}{ Lung cancer } \\
\hline Stage IIIB vs IIIA & $0.83 \pm 0.26$ & .001 & $69 \%$ \\
\hline No resection of lung cancer & $0.73 \pm 0.25$ & .004 & $55 \%$ \\
\hline \multicolumn{4}{|l|}{ Brain metastases } \\
\hline Older age at diagnosis of brain metastases & $0.038 \pm 0.0147$ & .009 & $68 \%$ \\
\hline Worse ECOG performance statust & $0.36 \pm 0.088$ & $<.0001$ & $93 \%$ \\
\hline Presence of other systemic metastases & $1.04 \pm 0.33$ & .002 & $53 \%$ \\
\hline \multicolumn{4}{|l|}{ Treatment of brain metastases } \\
\hline No resection of brain metastases & $1.64 \pm 0.36$ & $<.0001$ & $96 \%$ \\
\hline No stereotactic radiosurgery & $1.11 \pm 0.32$ & .0005 & \\
\hline Earlier date of treatment $\ddagger$ & $-0.34 \pm 0.140$ & .01 & $62 \%$ \\
\hline
\end{tabular}

Coefficients are \pm SE.

*Frequency of appearance in 1000 bootstrap analyses (see Methods section).

†ECOG classes 0 and 1 combined as class 0 , ECOG class 2 as class 1, and ECOG class 3 as class 2, squared transformation.

$\neq$ Derived as In [date of treatment (in years since June 1, 1980)], natural logarithmic transformation.

TABLE 2. Correlates of metastasectomy or stereotactic radiosurgery for brain metastases

\begin{tabular}{lccc}
\hline Correlate & Odds ratio & 95\% Cl & P value \\
\hline $\begin{array}{l}\text { Resection of lung cancer } \\
\text { Yes vs no }\end{array}$ & 4.0 & $1.3-13$ & .02 \\
\hline $\begin{array}{l}\text { ECOG performance status } \\
\quad \text { Per 1-pt increase }\end{array}$ & 0.26 & $0.10-0.68$ & .006 \\
$\begin{array}{l}\text { Other systemic metastases } \\
\quad \text { Absent vs present }\end{array}$ & 9.1 & $1.6-50$ & .01 \\
\hline $\begin{array}{l}\text { No. of brain metastases } \\
\text { Per 1-metastasis increase }\end{array}$ & 0.4 & $0.24-0.66$ & $<.001$ \\
\hline
\end{tabular}

Figure 3 illustrates four combinations of patients and treatment of brain metastases. Figure 3, A, demonstrates the clinically important survival benefit of metastasectomy or stereotactic radiosurgery relative to palliation for metachronous brain metastases in a patient with characteristics predictive of good survival. Figure 3, B, demonstrates the statistically significant but clinically unimportant survival benefit of metastasectomy or stereotactic radiosurgery relative to palliation for metachronous brain metastases in a patient with characteristics predictive of poor survival.

\section{Treatment Benefit or Patient Selection?}

Multivariable analysis of factors associated with metastasectomy or stereotactic radiosurgery demonstrated patient selection (Table 2 and Appendix B). Two propensitystratified groups addressed this patient selection (Table 3). Group 1 consisted predominantly of patients with characteristics predictive of good survival, most of whom were treated with metastasectomy or stereotactic radiotherapy. A small number received palliation. In contrast, group 2 was predominantly patients with characteristics predictive of poor survival, most of whom received palliation. A small number underwent metastasectomy or stereotactic radiotherapy. In both groups a survival advantage of metastasectomy or stereotactic radiosurgery with respect to palliation was demonstrated (Figure 4).

\section{Optimal Management Revisited}

Adding propensity score $(P=.3)$ to the multivariable analysis of death confirmed the survival benefit of metastasectomy $(P=.0001)$ or stereotactic radiosurgery $(P=$ $.0003)$ and thus the inferences concerning optimal management.

\section{Discussion \\ Optimal Management}

Patients with untreated NSCLC brain metastases die of progressive neurologic deterioration within 1 month. ${ }^{10}$ Survival and quality of life for patients with solitary brain metastases are superior when metastasectomy is included in the treatment. ${ }^{11}$ However, complete resection of the primary lung cancer and control of the primary and other distant extracranial metastatic sites determine outcome after brain metastasectomy (Appendices A and B).

Solitary brain metastasis has been a selection factor for metastasectomy; however, many patients have more than one brain metastasis. ${ }^{10,12}$ This and other selection factors have produced a literature of treatment of brain metastases in highly selected patients. Many readers attribute good survival to a given treatment; however, it may actually be due to patient selection. Thus, although it is intuitively apparent that some patients will benefit from aggressive treatment of their brain metastases, it remains unclear which patients should be treated and how. Optimal treatment of patients with NSCLC brain metastases cannot be generalized, because they are heterogeneous and treatment must be individually selected. 
TABLE 3. Comparisons within propensity-matched groups

\begin{tabular}{|c|c|c|c|c|c|c|}
\hline \multirow[b]{2}{*}{ Variable } & \multicolumn{3}{|c|}{ Group 1 (good survival) } & \multicolumn{3}{|c|}{ Group 2 (poor survival) } \\
\hline & $\begin{array}{l}\text { Palliation } \\
(n=6)\end{array}$ & $\begin{array}{l}\text { Treatment* }^{*} \\
(\mathrm{n}=\mathbf{4 0})\end{array}$ & $P$ value & $\begin{array}{l}\text { Palliation } \\
(\mathrm{n}=38)\end{array}$ & $\begin{array}{l}\text { Treatment* } \\
\quad(\mathrm{n}=7)\end{array}$ & $P$ value \\
\hline \multicolumn{7}{|l|}{ Demographics } \\
\hline Male $(\%)$ & 50 & 58 & .7 & 53 & 43 & .6 \\
\hline \multicolumn{7}{|l|}{ 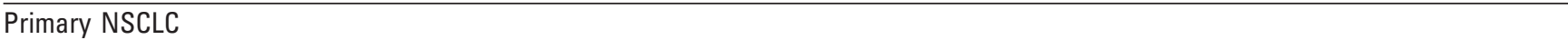 } \\
\hline \multicolumn{7}{|l|}{ Histologic type $(\%)$} \\
\hline Squamous cell & 33 & 28 & 6 & 24 & 43 & .16 \\
\hline Adenocarcinoma & 33 & 52 & & 66 & 29 & \\
\hline Large cell & 33 & 20 & & 11 & 29 & \\
\hline Stage IIIA (\%) & 50 & 55 & .8 & 63 & 43 & .3 \\
\hline \multicolumn{7}{|l|}{ Resection of lung cancer $(\%)$} \\
\hline None & 17 & 22 & .9 & 53 & 29 & .5 \\
\hline Segmentectomy and lobectomy & 50 & 40 & & 37 & 57 & \\
\hline Pneumonectomy & 33 & 38 & & 11 & 14 & \\
\hline \multicolumn{7}{|l|}{ Brain metastases } \\
\hline Disease-free interval (mo) & $8.6 \pm 5.5$ & $14.9 \pm 12.4$ & .2 & $10.6 \pm 8.4$ & $6.2 \pm 2.5$ & .17 \\
\hline Age at diagnosis $(y)$ & $61 \pm 6$ & $57 \pm 9$ & .4 & $62 \pm 10$ & $60 \pm 11$ & .6 \\
\hline \multicolumn{7}{|l|}{ ECOG performance status (\%) } \\
\hline 0 & 0 & 2 & .5 & 0 & 14 & .08 \\
\hline 1 & 100 & 82 & & 39 & 43 & \\
\hline 2 & 0 & 15 & & 42 & 43 & \\
\hline $3-4$ & 0 & 0 & & 18 & 0 & \\
\hline Primary NSCLC recurrence (\%) & 17 & 18 & .96 & 58 & 43 & .5 \\
\hline Other systemic metastases $(\%)$ & 17 & 5 & .3 & 26 & 14 & .5 \\
\hline \multicolumn{7}{|c|}{ Recursive partitioning analysis score (\%) } \\
\hline 1 & 50 & 62 & .6 & 5 & 14 & .4 \\
\hline 2 & 50 & 38 & & 58 & 71 & \\
\hline 3 & 0 & 0 & & 37 & 14 & \\
\hline No. of brain lesions (mean \pm SD) & $1.8 \pm 1.3$ & $1.5 \pm 0.8$ & .3 & $2.8 \pm 1.2$ & $2.6 \pm 1.1$ & .7 \\
\hline Unilateral brain metastases $(\%)$ & 83 & 92 & .5 & 47 & 29 & .4 \\
\hline
\end{tabular}

*Treatment was either metastasectomy or stereotactic radiosurgery.

One aspect of heterogeneity is stage of primary NSCLC. Neoadjuvant treatment of stage III NSCLC produces a unique cohort of patients who have the primary NSCLC controlled and survive to have brain metastases develop. ${ }^{3}$ Another aspect is the method of treatment of the brain metastases. Although no phase III trials comparing metastasectomy with stereotactic radiosurgery have been completed, our report and others have demonstrated similar survivals between these modalities. ${ }^{13-15}$ Only technical considerations (large metastasis and neurologic deterioration from mass effect) limit the use of stereotactic radiosurgery.

In this report, both metastasectomy and stereotactic radiosurgery produced a survival advantage relative to wholebrain radiation or symptomatic therapy (palliation). For the patient with characteristics predictive of poor survival, this advantage was statistically significant but clinically unimportant. Therefore, in such patients quality of life becomes the goal of therapy. Stereotactic radiosurgery spares the patient the trauma of metastasectomy and may provide the best palliation. Whole-brain radiation therapy should be reserved for patients who are not candidates for stereotactic radiosurgery.
For the patient with characteristics predictive of good survival-younger age (Appendix Figure A), previous resection of NSCLC, stage IIIA, good performance status at diagnosis of brain metastasis, and no other distant metastases-survival was better if cure rather than palliation was the goal. The "good-risk" patient is denied longterm survival if treated with palliative intent using only whole-brain radiation or symptomatic therapy. Because of equivalent survivals with metastasectomy and stereotactic radiosurgery in these patients and the lack of any phase III trials, there is uncertainty-even among ourselves-about which of these two modalities should be offered to which patient.

\section{Treatment Benefit or Treatment Selection?}

Lack of a management protocol produced variations in treatment and thus provided a spectrum of patient-therapy combinations. A patient with characteristics predictive of good survival was more likely to be selected for metastasectomy or stereotactic radiosurgery, whereas a patient with characteristics predictive of poor survival was more likely to be selected for whole-brain radiotherapy or symptomatic 

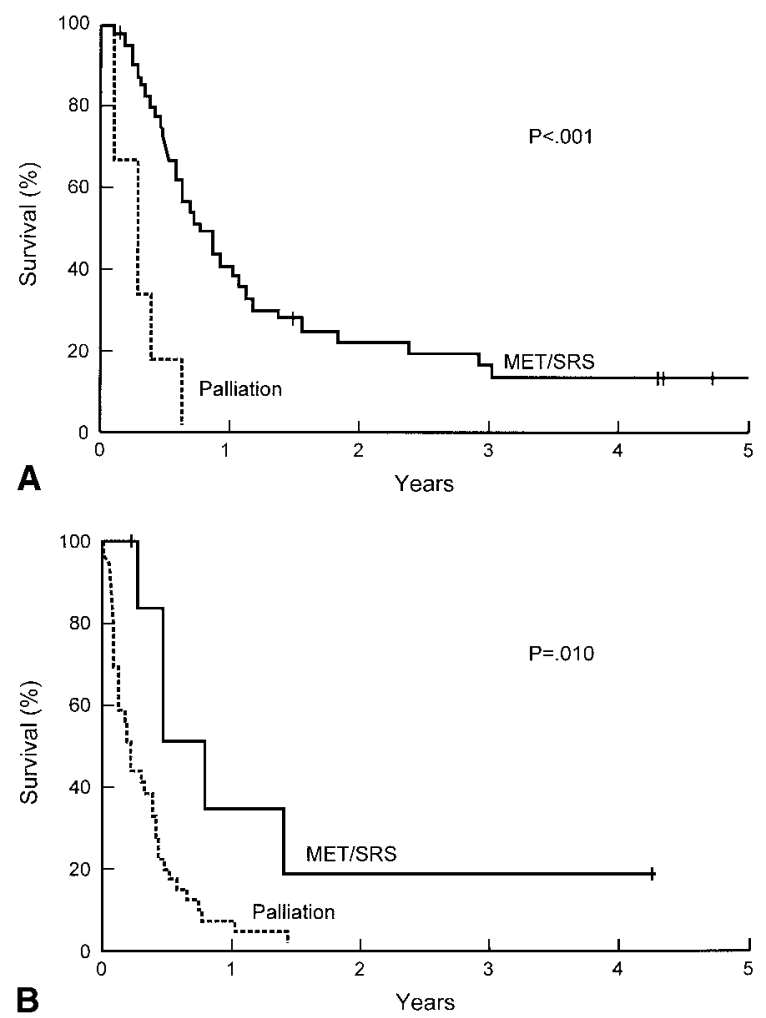

Figure 4. Survival of propensity-matched patients stratified by treatment of metachronous brain metastases. Figure format as in Figure 1. A, Group 1, patients at good risk. Survival estimates and numbers of patients remaining at risk at 6, 12, and 24 months were as follows: for metastasectomy and stereotactic radiosurgery (MET/SRS, solid line, $n=40), 74 \% \pm 7.0 \%(n=29), 40 \% \pm 8.0 \%$ ( $n=15)$, and $21 \% \pm 6.7 \%(n=7)$, respectively; and for palliation (broken line, $n=6), 17 \% \pm 15 \%(n=1), 0 \%(n=0)$, and $0 \%(n=$ $0)$, respectively. B, Group 2, patients at poor risk. Survival estimates and numbers of patients remaining at risk at 6, 12, and 24 months were as follows: for metastasectomy and stereotactic radiosurgery (MET/SRS, solid line, $n=7), 50 \% \pm 20 \%(n=3)$, $33 \% \pm 19 \%(n=2)$, and $17 \% \pm 15 \%(n=1)$, respectively; and for palliation (broken line, $n=38$ ), $18 \% \pm 6.3 \%(n=7), 5 \% \pm 3.6 \%$ $(n=2)$, and $0 \%(n=0)$, respectively.

therapy. Propensity score methodology was developed to address nonrandomized comparisons in which therapy has not been applied either randomly or systematically. 7,9

Use of the propensity score in this study allowed patient selection to be separated from treatment effect. We used propensity scores in two ways in the analysis. First, we found in propensity-matched patients a uniform benefit of treating metachronous brain metastases with metastasectomy or stereotactic radiosurgery. Second, we introduced the propensity score into the multivariable analysis of optimal treatment and confirmed the benefit of metastasectomy or stereotactic radiosurgery. Both analyses demon- strated a clinically significant benefit only for the good-risk patient. ${ }^{9}$

\section{Limitations}

This was a small, single-institution clinical experience. The study required that all patients survive to develop brain metastases, making interim death a selection factor. Patients were selected for treatment by clinical criteria; treatment was administered in a nonrandom fashion. To limit the influence of nonrandomization, we used propensity scores as described previously. Despite our use of 10 variables to develop the score and produce well-matched groups, the propensity score did not match for unrecorded variables that may have influenced selection.

\section{Conclusion}

Younger patients with resected stage IIIA NSCLC who have isolated metachronous brain metastases and good performance status do best when treated with metastasectomy or stereotactic radiosurgery. Survival benefit is a brain treatment effect and not the result of selecting the best candidates for aggressive therapy.

\section{References}

1. MacGee EE. Surgical treatment of cerebral metastases from lung cancer: the effect on quality and duration of survival. J Neurosurg. 1971;35:416-20.

2. Magilligan DJ Jr, Rogers JS, Knighton RS, Davila JC. Pulmonary neoplasm with solitary cerebral metastasis: results of combined excision. J Thorac Cardiovasc Surg. 1976;72:690-8.

3. Law A, Karp DD, Dipetrillo T, Daly BT. Emergence of increased cerebral metastasis after high-dose preoperative radiotherapy with chemotherapy in patients with locally advanced nonsmall cell lung carcinoma. Cancer. 2001;92:160-4.

4. Andre F, Grunenwald D, Pujol JL, Girard P, Dujon A, Brouchet L, et al. Patterns of relapse of N2 nonsmall-cell lung carcinoma patients treated with preoperative chemotherapy: should prophylactic cranial irradiation be reconsidered? Cancer. 2001;91:2394-400.

5. Blackstone EH, Naftel DC, Turner ME Jr. The decomposition of time-varying hazard into phases, each incorporating a separate stream of concomitant information. J Am Stat Assoc. 1986;81:615-24.

6. Breiman L. Bagging predictors. Machine Learning. 1996;26:123-40.

7. Rosenbaum PR, Rubin DB. The central role of the propensity score in observational studies for causal effects. Biometrika. 1983;70:41-55.

8. Gaspar L, Scott C, Rotman M, Asbell S, Phillips T, Wasserman T, et al. Recursive partitioning analysis (RPA) of prognostic factors in three Radiation Therapy Oncology Group (RTOG) brain metastases trials. Int J Radiat Oncol Biol Phys. 1997;37:745-51.

9. Drake C, Fisher L. Prognostic models and the propensity score. Int J Epidemiol. 1995;24:183-7.

10. Kelly K, Bunn PA Jr. Is it time to reevaluate our approach to the treatment of brain metastases in patients with non-small cell lung cancer? Lung Cancer. 1998;20:85-91.

11. Patchell RA, Tibbs PA, Walsh JW, Dempsey RJ, Maruyama Y, Kryscio RJ, et al. A randomized trial of surgery in the treatment of single metastases to the brain. N Engl J Med. 1990;322:494-500.

12. Wronski M, Arbit E, Burt M, Galicich JH. Survival after surgical treatment of brain metastases from lung cancer: a follow-up study of 231 patients treated between 1976 and 1991. J Neurosurg. 1995;83: 605-16.

13. Flickinger JC, Kondziolka D, Lunsford LD, Coffey RJ, Goodman ML, Shaw EG, et al. A multi-institutional experience with stereotactic 
radiosurgery for solitary brain metastasis. Int $J$ Radiat Oncol Biol Phys. 1994;28:797-802.

14. Alexander E, Moriarty TM, Davis RB, Wen PY, Fine HA, Black PM, et al. Stereotactic radiosurgery for the definitive, noninvasive treatment of brain metastases. J Natl Cancer Inst. 1995;87:34-40.

15. Kim YS, Kondziolka D, Flickinger JC, Lunsford LD. Stereotactic radiosurgery for patients with nonsmall cell lung carcinoma metastatic to the brain. Cancer. 1997;80:2075-83.

\section{Appendix A}

Impact of Stage III Non-Small Cell Lung Cancer and Its Treatment on Metachronous Brain Metastases

In this nonrandomized clinical experience, characteristics and treatment of the primary lung cancer varied. These factors may have influenced both development and treatment of metachronous brain metastases.

\section{Stage III Non-Small Cell Lung Cancer}

Time interval between treatment of NSCLC and diagnosis of brain metastases was highly variable. According to multivariable analysis, longer disease-free intervals were seen in men (hazard ratio $0.55,95 \%$ CI $0.35-0.86, P=.009$ ), for squamous cell carcinoma versus large cell undifferentiated carcinoma (hazard ratio 0.47 , 95\% CI 0.24-0.91, $P=.02$ ), for adenocarcinoma versus large cell carcinoma (hazard ratio $0.58,95 \%$ CI $0.33-1.03, P=.06$ ), and in patients who had undergone pneumonectomy (hazard ratio 0.61, 95\% CI 0.37-1.0, $P=.05$ ).

Patients who had undergone pneumonectomy had fewer brain metastases ( $n=18 / 22$ [82\%] with 1 or 2 brain metastases) than did those who had undergone segmentectomy or lobectomy $(\mathrm{n}=$ 20/37 [54\%] with 1 or 2 brain metastases, $P=.03$ ). Unilateral brain metastases were present in 21 patients who had undergone pneumonectomy (95\%) and in only 24 who had undergone segmentectomy or lobectomy $(65 \%, P=.008)$. Bilateral brain metastases were more common among patients who did not undergo resection of NSCLC $(\mathrm{n}=15 / 32,47 \%)$ than among patients who had resection ( $\mathrm{n}=14 / 59,24 \%, P=.02$ ). However, the numbers of brain metastases in these two groups were similar $(P=.6)$. Other distant metastases were more frequent at the time of diagnosis of brain metastases among patients who had undergone resection of $\operatorname{NSCLC~}(\mathrm{n}=12 / 59,20 \%$, vs $\mathrm{n}=2 / 32,6 \%, P=.08)$.

\section{Treatment of Stage III Non-Small Cell Lung Cancer}

Patients who had undergone resection only of NSCLC had a similar number of brain metastases $(n=8 / 11$ [73\%] with 1 or 2 brain metastases) to those who had also undergone postoperative adjuvant or neoadjuvant therapy ( $\mathrm{n}=30 / 48$ [62\%] with 1 or 2 brain metastases, $P=.5$ ). These two groups of patients were similar in demographic characteristics, tumor factors, and treatment of brain metastases (Appendix Table B).

Men were more likely to have undergone pneumonectomy $(\mathrm{n}=$ $14 / 22,64 \%$ ) than lobectomy or segmentectomy ( $\mathrm{n}=15 / 37,41 \%$, $P=.09)$.

Sites of active tumor found at death differed with treatment of NSCLC. Among 32 patients without resection, sites of cancer were locoregional (lung) in 25 cases (78\%), brain in $21(66 \%)$, and other distant metastases in 9 (28\%). In contrast, among 32 patients with segmentectomy or lobectomy, sites of cancer were locoregional in 14 cases $(44 \%)$, brain in $18(56 \%)$, and other distant metastases in
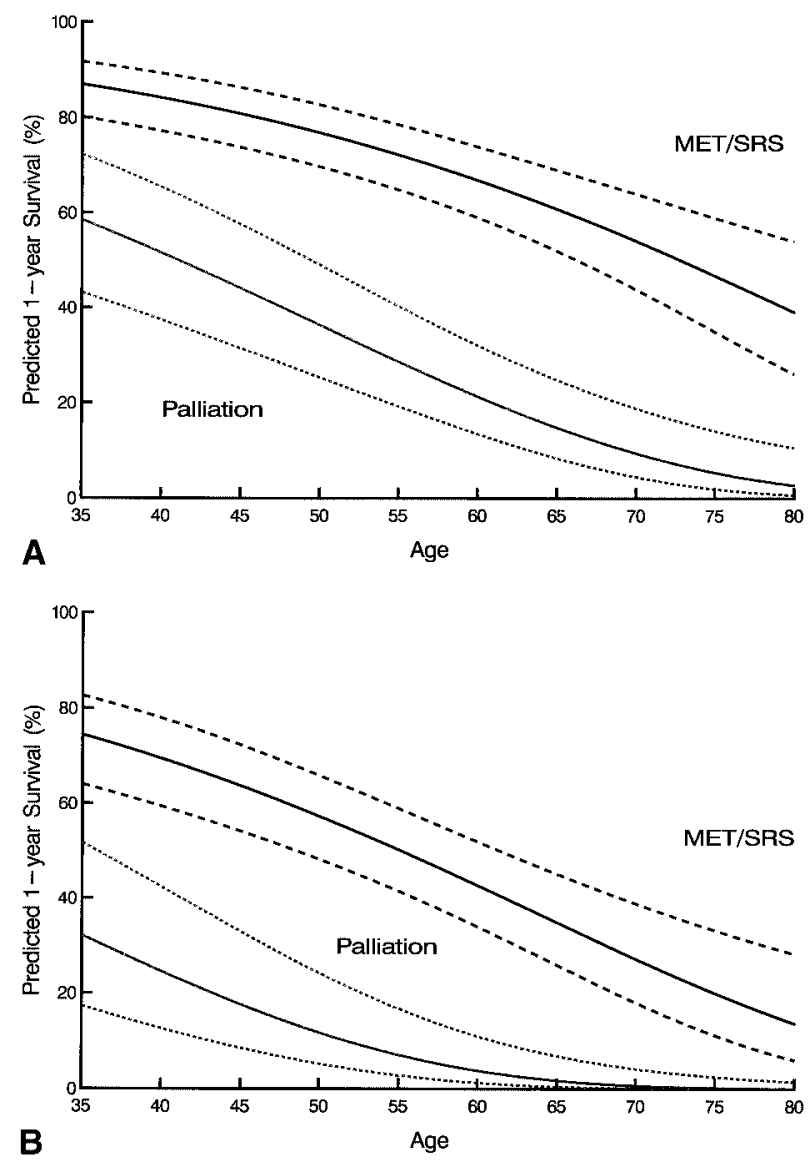

Appendix Figure A. Predicted 1-year survival according to age of patient having undergone pneumonectomy, with ECOG 0 to 1 and no other systemic metastases at time of diagnosis of metachronous brain metastases. Multivariable equation has been solved for either metastasectomy or stereotactic radiosurgery (MET/SRS) or palliation. Dashed lines represent $68 \%$ confidence intervals. A, Stage IIIA NSCLC. B, Stage IIIB NSCLC.

$7(22 \%)$. Among 19 patients with pneumonectomy, sites of cancer were locoregional in 7 cases (37\%), brain in $8(42 \%)$, and other distant metastases in $8(42 \%)$.

\section{Appendix B}

Factors Influencing Treatment of Brain Metastases

No clear pattern of treatment of brain metastases emerged over time. However, treatment varied with age, with younger patients likely to undergo metastasectomy, somewhat older patients likely to undergo stereotactic radiotherapy, and the oldest patients likely to undergo whole-brain radiation therapy (Appendix Table A).

Treatment also varied with disease-free interval. Disease-free intervals were longest for patients who had metastasectomy, shorter for those receiving stereotactic radiosurgery, and shortest for those receiving palliation.

Treatment varied with the number of brain metastases. Two or fewer brain metastases were seen in $87 \%$ of patients who had metastasectomy, in $75 \%$ who had stereotactic radiosurgery, and in 
Appendix Table A. Treatment of brain metastases

\begin{tabular}{|c|c|c|c|c|c|}
\hline Variable/group & $\begin{array}{l}\text { Palliation* } \\
\text { (n = 44) }\end{array}$ & $\begin{array}{l}\text { Metastasectomy } \\
\quad(\mathrm{n}=23)\end{array}$ & $\begin{array}{l}\text { Stereotactic } \\
\text { radiosurgery } \\
(n=24)\end{array}$ & $\begin{array}{c}P \text { for palliation vs } \\
\text { metastasectomy and } \\
\text { stereotactic radiosurgery }\end{array}$ & $\begin{array}{c}P \text { for metastasectomy vs } \\
\text { stereotactic } \\
\text { radiosurgery }\end{array}$ \\
\hline \multicolumn{6}{|l|}{ Demographics } \\
\hline Male (no.) & $23(52 \%)$ & $14(61 \%)$ & $12(50 \%)$ & .7 & .4 \\
\hline \multicolumn{6}{|l|}{ Primary NSCLC } \\
\hline \multicolumn{6}{|l|}{ Histologic type (no.) } \\
\hline Squamous cell & $11(25 \%)$ & $10(44 \%)$ & $4(17 \%)$ & .09 & .04 \\
\hline Adenocarcinoma & $27(61 \%)$ & $7(30 \%)$ & $16(67 \%)$ & & \\
\hline Large cell & $6(14 \%)$ & $6(26 \%)$ & $4(17 \%)$ & & \\
\hline Stage IIIA (no.) & $27(61 \%)$ & $11(48 \%)$ & $14(58 \%)$ & 6 & .5 \\
\hline \multicolumn{6}{|l|}{ Resection of lung cancer (no.) } \\
\hline None & $21(48 \%)$ & $4(17 \%)$ & $7(29 \%)$ & .07 & .6 \\
\hline Segmentectomy or lobectomy & $17(39 \%)$ & $11(48 \%)$ & $9(38 \%)$ & & \\
\hline Pneumonectomy & $6(14 \%)$ & $8(35 \%)$ & $8(33 \%)$ & & \\
\hline \multicolumn{6}{|l|}{ Treatment of lung cancer (no.) } \\
\hline Palliationt & $21(48 \%)$ & $4(17 \%)$ & $7(29 \%)$ & .13 & .5 \\
\hline Resection only & $4(9 \%)$ & $3(13 \%)$ & $4(17 \%)$ & & \\
\hline Resection with adjuvant therapy & $19(43 \%)$ & $16(70 \%)$ & $13(54 \%)$ & & \\
\hline \multicolumn{6}{|l|}{ Brain metastases } \\
\hline Disease-free interval (mo) & $10.4 \pm 8.0$ & $16.0 \pm 14.0$ & $11.3 \pm 9.1$ & .10 & .18 \\
\hline Age at diagnosis $(y)$ & $62 \pm 10$ & $56 \pm 10$ & $59 \pm 9$ & .04 & .2 \\
\hline \multicolumn{6}{|l|}{ ECOG performance status (no.) } \\
\hline $0-1$ & $21(48 \%)$ & $19(83 \%)$ & $19(79 \%)$ & .008 & .8 \\
\hline 2 & $16(36 \%)$ & $4(17 \%)$ & $5(21 \%)$ & & \\
\hline $3-4$ & $7(16 \%)$ & $0(0 \%)$ & $0(0 \%)$ & & \\
\hline Primary NSCLC recurrence (no.) & $23(52 \%)$ & $8(35 \%)$ & $2(8 \%)$ & .002 & .03 \\
\hline Other systemic metastases (no.) & $11(25 \%)$ & $2(9 \%)$ & $1(4 \%)$ & .04 & .55 \\
\hline \multicolumn{6}{|c|}{ Recursive partitioning analysis score (no.) } \\
\hline 1 & $5(11 \%)$ & $11(48 \%)$ & $15(62 \%)$ & $<.001$ & .4 \\
\hline 2 & $25(57 \%)$ & $11(48 \%)$ & $9(38 \%)$ & & \\
\hline 3 & $14(32 \%)$ & $1(4 \%)$ & $0(0 \%)$ & & \\
\hline No. of metastases, 1 or 2 (no.) & $19(43 \%)$ & $20(87 \%)$ & $18(75 \%)$ & $<.001$ & .3 \\
\hline Unilateral brain metastases (no.) & $23(52 \%)$ & $20(87 \%)$ & $19(79 \%)$ & .006 & .5 \\
\hline
\end{tabular}

*Palliation for brain metastases consisted of 1 patient who received no treatment and 43 who received whole-brain radiotherapy alone.

tPalliation for lung cancer consisted of 3 patients who received no treatment and 29 who received chemoradiotherapy.

$43 \%$ who had palliation. Unilateral brain metastases were more likely to be treated with metastasectomy $(87 \%)$ or stereotactic radiosurgery $(79 \%)$ than with palliation $(52 \%)$. NSCLC was controlled in $92 \%$ of patients receiving stereotactic radiosurgery, in $65 \%$ undergoing metastasectomy, and in $48 \%$ receiving palliation. Other distant metastases were present in $4 \%$ of patients receiving stereotactic radiosurgery and in $9 \%$ undergoing metastasectomy, but in $25 \%$ receiving palliation.

\section{Discussion}

Dr Raphael Bueno (Boston, Mass). Moazami and colleagues, in this comprehensive manuscript from the Cleveland Clinic, evaluated the treatment of metachronous brain metastases in patients with locally advanced lung cancer. They found that younger patients with stage IIIA lung cancer who had previously been treated with aggressive locoregional control of the primary cancer derived substantial survival benefit from an aggressive treatment of the brain metastases. They further found that survival after stereotactic radiosurgery and metastasectomy might be equivalent for patients with metastases smaller than $4 \mathrm{~cm}$ and better than after palliative radiation. Their results also suggest that the brain is occasionally the only site of metastasis in patients with resected N2-positive stage IIIA lung cancer, a finding that is consistent with other observations in the field of lung cancer as well as with our recent analysis of patients treated for stage IIIA lung cancer with multimodal therapy. The take-home message from this article is that it is reasonable to be aggressive when treating patients with good performance and locoregional control of the lung cancer who have isolated brain metastases.

I would like to pose a few questions. First, please tell us more about the long-term survivors. Were they all young patients with resected stage IIIA disease and single brain metastases, or are there some older ones in the group? Also, perhaps we should use functional status rather than age in the treatment algorithm, because even though posttreatment survival in your series was a little worse among elderly patients, there is no other effective therapy.

Second, do you currently ever treat patients with stage IIIB lung cancer with aggressive brain resection since the analysis of your results? If so, why? 
Appendix Table B. Resection versus resection and adjuvant therapy for the primary NSCLC

\begin{tabular}{|c|c|c|c|}
\hline & $\begin{array}{l}\text { Resection alone } \\
\quad(n=11)\end{array}$ & $\begin{array}{l}\text { Resection plus adjuvant } \\
\text { therapy }(n=48)\end{array}$ & $P$ value \\
\hline \multicolumn{4}{|l|}{ Demographics } \\
\hline Male (no.) & $7(64 \%)$ & $22(46 \%)$ & .3 \\
\hline \multicolumn{4}{|l|}{ Primary NSCLC } \\
\hline \multicolumn{4}{|l|}{ Histologic type (no.) } \\
\hline Squamous cell & $4(36 \%)$ & $10(21 \%)$ & .15 \\
\hline Adenocarcinoma & $7(64 \%)$ & $26(54 \%)$ & \\
\hline Large cell & $0(0 \%)$ & $12(25 \%)$ & \\
\hline Stage IIIA (no.) & $7(64 \%)$ & $29(60 \%)$ & .8 \\
\hline Resection, pneumonectomy (no.) & $5(45 \%)$ & $17(35 \%)$ & .5 \\
\hline \multicolumn{4}{|l|}{ Brain metastases } \\
\hline Disease-free interval (mo) & $13.3 \pm 16.7$ & $11.9 \pm 8.1$ & .7 \\
\hline Age at diagnosis of brain metastases (y) & $60 \pm 11$ & $58 \pm 10$ & 6 \\
\hline \multicolumn{4}{|l|}{ ECOG performance status (no.) } \\
\hline $0-1$ & $9(82 \%)$ & $32(67 \%)$ & .3 \\
\hline 2 & $1(9 \%)$ & $14(29 \%)$ & \\
\hline $3-4$ & $1(9 \%)$ & $2(4 \%)$ & \\
\hline Control of primary NSCLC (no.) & $8(73 \%)$ & $38(79 \%)$ & 6 \\
\hline Other systemic metastases (no.) & $3(27 \%)$ & $9(19 \%)$ & .5 \\
\hline \multicolumn{4}{|l|}{ Recursive partitioning analysis score (no.) } \\
\hline 1 & $4(36 \%)$ & $20(42 \%)$ & .9 \\
\hline 2 & $5(45 \%)$ & $21(44 \%)$ & \\
\hline 3 & $2(18 \%)$ & $7(15 \%)$ & \\
\hline No. of metastases 1 or 2 (no.) & $8(73 \%)$ & $30(62 \%)$ & .5 \\
\hline Unilateral brain metastases (no.) & $8(73 \%)$ & $37(77 \%)$ & .8 \\
\hline \multicolumn{4}{|l|}{ Treatment of brain metastases (no.) } \\
\hline Palliation & $4(36 \%)$ & $19(40 \%)$ & .8 \\
\hline Metastasectomy & $3(27 \%)$ & $16(33 \%)$ & \\
\hline Stereotactic radiosurgery & $4(36 \%)$ & $13(27 \%)$ & \\
\hline
\end{tabular}

Third, what is your denominator for this study, first in terms of all patients treated at your institution with stage III lung cancer and second in terms of all patients with stage III cancer who had distant metastases develop? How many of those are just in the brain?

Fourth, are isolated brain metastases the most common first site of failure in your institution for stage III lung cancer? We and others have noted that, with a rate greater than $50 \%$ in our series. If that is the case, should we consider prophylactic whole-brain radiation for patients who undergo resection of stage IIIA lung cancer?

Finally, how do you currently treat a patient with a T1 N2 M1 NSCLC whose only synchronous metastatic focus is an isolated 2 $\mathrm{cm}$ brain metastasis?

Dr Moazami. Dr Bueno, thank you for your comments. I will try to address your questions in the order that you have addressed them.

You asked about the long-term survivors in this study. We had a total of 91 patients, 8 of whom were alive at the end of our follow-up period, which was completed in February 2001. Mean follow-up of the patients was 36 months. Their mean age was 48 years (range 36-61 years). As you suggested, the patients in the survival group actually correlated well with the variables that we identified for best survival; that is, they were generally younger patients with good performance status and controlled stage IIIA disease in whom we had not detected any distant metastases other than in the brain.
Your second question was whether we would aggressively treat the brain lesion in a patient with stage IIIB given the results of our data. I think that in general the results of all retrospective studies such as this provide us with some guidelines, but obviously treatment needs to be considered on a patient-to-patient basis. For a patient with stage IIIB NSCLC that has been aggressively treated with good locoregional control, we believe that it is reasonable to proceed with aggressive treatment of an isolated brain lesion with either resection or perhaps stereotactic radiosurgery. Future studies should clarify whether stereotactic radiosurgery or metastasectomy is the better treatment. Again, one of the purposes of this extensive analysis was to show that, regardless of other factors, metastasectomy or stereotactic radiosurgery does confer a survival benefit on these patients.

You asked about the denominator of the study. We did an extensive review of all the medical records at the Cleveland Clinic during this 10-year period to try to select all the patients diagnosed with stage III NSCLC. The total number of patients was 891 . Of those, we selected only the patients who had metachronous brain metastases develop, which we considered as 3 months from the time of diagnosis of lung cancer. This gave us the numerator, which was 91 patients.

You asked whether we believe that the brain is commonly the isolated site of failure and whether prophylactic whole-brain radiation therapy should be given to this patient population. We 
similarly found that in our study group, $46 \%$ of these patients had an isolated brain metastasis. Whether we should prophylactically radiate the brain has not been clarified in the literature. Most of the studies that I am aware of have not shown any benefit of prophylactic whole-brain radiation in any of the populations, with the possible exception of those with small cell cancer.

Finally, you asked about a patient with a stage IIIA disease, T1 N2 M1, who has only a synchronous brain metastasis. Obviously, the study was not meant to address synchronous brain metastases. However, we believe that once an aggressive approach to locoregional control is established, it is also reasonable to proceed with an aggressive treatment regimen for the brain. How to proceed in treating a patient like this depends on the variables we have discussed, including performance status.

Dr Robert J. Ginsberg (Toronto, Ontario, Canada). You had 8 long-term survivors. How many had 2 or more brain metastases?

Dr Moazami. Of the 8 survivors, 5 had 1 brain metastasis, 1 had 2 metastases, and 2 had 3 metastases.

Dr Ginsberg. Why was it not one of your conclusions that you should limit this aggressive therapy to single metastases?

Dr Moazami. Well, there was a sharp cutoff in survival when we divided the patients according to 1 to 2 brain metastases versus 3 or more. In our conclusion we stated that the best candidates are the ones that need to be aggressively treated. In this best profile that we constructed, one factor was the presence of 1 to 2 metastases.

Dr Ginsberg. But you did not differentiate between 1 versus 2?
Dr Moazami. There was no difference in survival between patients with 1 or 2 brain metastases. Predicted Kaplan-Meier median survival was 6.3 months for 1 brain metastasis and 7.3 months for 2 brain metastases.

Dr Ginsberg. Were any patients with 2 metastases treated with surgery?

Dr Moazami. Of 15 patients with 2 brain metastases, 7 had palliative whole-brain radiotherapy, 4 had stereotactic radiotherapy, and 4 had resection with whole-brain radiotherapy.

Dr Douglas E. Wood (Seattle, Wash). How do you feel that you corrected for selection bias in the second half of your analysis, that is, that the factors you identified, in vanishingly smaller numbers, represent a treatment effect as opposed to a selection effect of patients? And do you believe that the success in the aggressively treated patients was due to systemic tumor control or improved palliation within the brain?

Dr Moazami. The issue of selection bias was a concern. We tried to address it as best we could by using the statistical method of propensity matching. This allowed us to assign a propensity score on the basis of the 10 variables and match the patients. It was our best statistical tool to determine from this retrospective data analysis whether the treatment was effective. Prospective, randomized studies are another way to address such questions.

The data suggest that the patients who were aggressively treated had control both locoregionally and in the brain. Most failures were at distant sites.

\section{Targeted}

The Journal of Thoracic and Cardiovascular Surgery gives you two tables of contents.

The condensed table of contents tells you at a glance what topics and authors are presented each month. The expanded table of contents gives you a brief abstract of each article. You select only those articles of most interest to you for further reading. 